\title{
Tamoxifen-induced Nonalcoholic Steatohepatitis in Breast Cancer Patients Treated with Adjuvant Tamoxifen
}

\author{
Yoshihisa Nemoto, Toshiji Saibara, Yasuhiro Ogawa*, Ting Zhang, Nan Xu, Masafumi Ono, Naoaki Akisawa, \\ Shinji IwASAKI, Takashi MAEDA and Saburo ONISHI
}

\begin{abstract}
Objective The benefits of 5-year tamoxifen administration for patients with estrogen receptor-positive breast cancer undoubtedly outweigh the risk of any adverse effects. Massive hepatic steatosis is an example of its side effects. Here we show risk factors for the development of massive hepatic steatosis and describe a representative clinical course of these patients treated with fibrates for the first time.

Methods Computed tomography (CT) numbers of the liver and spleen were measured and correlated to body mass index (BMI) and pregnanetriol/pregnanediol ratio in urine $\left(\mathbf{P}_{3} / \mathbf{P}_{2}\right.$ ratio $)$.

Patients We enrolled 56 premenopausal women treated with breast conservation treatment. They received oral tamoxifen (40 mg/day for 2 to 3 years) as adjuvant endocrine therapy with systemic chemotherapy.

Results Serum estradiol level in 48 of 56 patients treated with tamoxifen was less than $10 \mathrm{pg} / \mathrm{ml}$. The ratio of hepatic CT number to splenic CT number $<0.9$ was related to increased BMI ( $>23.6 \mathrm{~kg} / \mathrm{sqm})$ and reduced $\mathrm{P}_{3} / \mathrm{P}_{2}$ ratio $(<\mathbf{1})$. Fibrates are potent enough to improve hepatic steatosis in tamoxifen-induced hepatic steatosis.

Conclusion $\mathbf{P}_{3} / \mathbf{P}_{2}$ ratio $<1$ was related to sufficient blockade of estrogen receptor proven by the development of massive hepatic steatosis. Therefore, we propose that tamoxifen is not a mere antagonist of estrogen, but it may also suppress estrogen synthesis when estrogen receptor is blocked sufficiently. The undetectable level of serum estrogen in tamoxifen-treated premenopausal women may partially explain why tamoxifen was so effective in estrogen receptor-positive early breast cancer.

(Internal Medicine 41: 345-350, 2002)
\end{abstract}

Key words: estrogen, CYP17 $\alpha$, fatty liver

\section{Introduction}

Tamoxifen is used worldwide as an antiestrogenic agent in the adjuvant therapy for women with estrogen receptor-positive early breast cancer (1). However, in patients treated with tamoxifen, endometrial cancer occurs more often than in the controls (2) and severe hyperlipidemia is observed occasionally (3). These findings are probably related to the intrinsic agonistic estrogenic effects of tamoxifen, but, to our knowledge, there has been no report of lipid metabolism dysfunction related to the antagonistic effects of tamoxifen which could be more frequent in adjuvant tamoxifen since tamoxifen is a potent antagonist of estrogen.

Recently we reported massive hepatic steatosis in one-third of non-obese breast cancer patients as a result of exposure to tamoxifen and some of these patients even developed nonalcoholic steatohepatitis (NASH) $(4,5)$. The extent of hepatic steatosis observed in adjuvant tamoxifen is so severe that computed tomography (CT) can easily detect hepatic steatosis; such severe hepatic steatosis is very rare in non-obese individuals (6). Fatty changes of the liver could be related to the antagonistic effects of tamoxifen on estrogen receptor, because massive hepatic steatosis spontaneously develops in aromatasedeficient (ArKO) mice (7), which lack intrinsic estrogen production (8). ArKO mice are severely defective in fatty acid $\beta$ oxidation and accumulate a large amount of triglycerides in hepatocytes, while the administration of extrinsic estrogen easily abrogates the development of such changes in the liver. These clinical and laboratory observations suggest that estrogen is implicated in constitutive hepatic lipid metabolism and sufficient blockade of estrogen-mediated pathways in the liver may cause massive hepatic steatosis and NASH. Here we show a possible mechanism why tamoxifen is so potent to antagonize the estrogen receptor-mediated signaling.

In the recent five years NASH has become the second or third commonest liver disease in outpatient hepatology practice in North America (9). This resulted in a debate whether hepatic steatosis, which is easily defined by the liver/spleen ratio of computed tomography values in Hounsfield units $<0.9$ (6), is an "innocent bystander or guilty party" in NASH (10).

From the Department of Internal Medicine and *the Department of Radiology, Kochi Medical School, Nankoku

Received for publication October 12, 2001; Accepted for publication January 16, 2002

Reprint requests should be addressed to Dr. Toshiji Saibara, the Department of Internal Medicine, Kochi Medical School, Nankoku 783-8505 
As liver diseases have not been widely appreciated as a lifethreatening complication of obesity, hepatic steatosis has been regarded to be an innocent bystander of NASH. However, evidence implying obesity as a risk for liver diseases has been accumulating. For example, liver cirrhosis is approximately six times more prevalent in obese individuals than in the general population and obesity increases the risk for liver cirrhosis (11) and gradual progression from hepatic steatosis to NASH and eventually to cirrhosis is supported by epidemiologic evidence (12). Thus, a consensus about NASH was provided recently: i.e., hepatic steatosis is regarded as a risk of NASH and a "second hit" capable of inducing necrosis, inflammation and fibrosis in the liver is required for the development of NASH since most patients with hepatic steatosis do not develop liver cirrhosis $(9,13,14)$. An exposure to endotoxin/bacterial lipopolysaccharides, iron overload, and accumulation of long chain and very long chain fatty acids were suggested as candidates for the "second hit" $(9,11,13)$.

Although many adverse effects have been reported (15), undoubtedly adjuvant tamoxifen outweighs the risks of any adverse effects. Therefore, we need to find ways for refraining from discontinuation of adjuvant tamoxifen. Tamoxifen-induced massive hepatic steatosis and NASH are frequent, but little has been reported about clinical features of NASH $(4,16$, 17) though it could progress to liver cirrhosis latently (12). Here, we described the representative clinical features of tamoxifeninduced NASH and suggest the administration of a peroxisome proliferator as one possible compensatory approach to prevent the progression of NASH (18) since administration of bezafibrate successfully prevented ArKO mice from developing massive hepatic steatosis (19).

\section{Materials and Methods}

\section{Patients}

We enrolled 56 women who were treated with breast conservation treatment (BCT) with permission from the Institutional Human Investigations Committee. They all received oral tamoxifen ( $40 \mathrm{mg} / \mathrm{day}$ for 2 to 3 years) as adjuvant endocrine therapy for BCT with systemic chemotherapy (cyclophosphamide, pirarubicin, 5-fluorouracil: CAF therapy) (20). Their body weight remained unchanged during adjuvant tamoxifen (BMI before treatment vs. during treatment: $22.9+/-2.8 \mathrm{~kg} / \mathrm{sqm}$ vs. $22.5+/-2.9 \mathrm{~kg} / \mathrm{sqm})$.

\section{Computed tomography (CT)}

We evaluated the CT number (Hounsfield units) of the liver and spleen each year before and after administration of tamoxifen. The CT scans of all patients were obtained with a GE CT9800 (General Electric, USA) and a helical-CT Proceed (GE-Yokogawa Medical Systems, Tokyo) with 10-mm thickness and 10-mm interval. Mean CT numbers of the liver and spleen were determined in the parenchyma of the right and left lobe of the liver and a similar area of the spleen, avoiding blood vessels, artifacts or heterogeneous areas. The criterion of diagnosis for hepatic steatosis was a ratio of CT numbers of the liver to those of the spleen (liver/spleen ratio) of less than 0.9 as described previously (6). The degree of hepatic steatosis is categorized as follows: severe (liver spleen ratio $<0$ ), moderate $(0-<0.5)$ or mild $(0.5-<0.9)$. CT findings are compared with body mass index (BMI) and the ratio of pregnanetriol $\left(\mathrm{P}_{3}\right)$ concentration to pregnanediol $\left(\mathrm{P}_{2}\right)$ concentration in urine $\left(\mathrm{P}_{3} / \mathrm{P}_{2}\right.$ ratio).

\section{Clinical course}

Bezafibrate ( $400 \mathrm{mg} /$ day) was administered to 10 patients with histologically proven tamoxifen-induced nonalcoholic steatohepatitis (NASH). Beneficial effects of fibrates on tamoxifen-induced hepatic steatosis were proven by sequential CT examination.

\section{Statistical analysis}

Kruskal-Wallis test and Fisher's exact test were used in the statistical analysis.

\section{Results}

\section{Computed tomography:}

Sequential changes of CT in one patient who rapidly developed severe hepatic steatosis are shown in Fig. 1. CT demonstrated hepatic steatosis in 19 of 56 patients as we have described previously $(4,5)$. Nineteen patients developed hepatic steatosis within the first two years, while the other 37 patients $(\mathrm{BMI}=21.5+/-2.6 \mathrm{~kg} / \mathrm{sqm}$, mean and standard deviation) have not developed hepatic steatosis at the time of examination. As there was no significant difference in inverse values of body weight between patients with hepatic steatosis and patients without hepatic steatosis $(0.0189+/-0.0028$ vs. $0.0182+/-$ $0.0027 \mathrm{~kg}^{-1}$, respectively) (Fig. 2), we may be able to exclude the possibility that the development of hepatic steatosis is due to excessive administration of tamoxifen.

\section{The degree of hepatic steatosis}

The degree of hepatic steatosis was mild in 12 cases, moderate in 5 cases and severe in 2 cases. BMI of these groups were $25.1+/-2.7,22.6+/-2.4$ and $24.6+/-0.7 \mathrm{~kg} / \mathrm{sqm}$, respectively. Fisher's exact test revealed that BMI $>23.6 \mathrm{~kg} / \mathrm{sqm}$ was related to a liver/spleen ratio of $<0.9$ as shown Fig. $3(\mathrm{p}<0.0001)$. These observations confirmed that the cut-off value of 23.6 in BMI is excellent for distinguishing two distinctive patterns of hepatic response to adjuvant tamoxifen therapy.

Serum estradiol level was $<10 \mathrm{pg} / \mathrm{ml}$ in all patients with a liver/spleen ratio of $<0.9$, but it was $<10 \mathrm{pg} / \mathrm{ml}$ in 48 of 56 patients. Therefore, the estradiol level was not useful enough to predict the progression of hepatic steatosis. The $\mathrm{P}_{3} / \mathrm{P}_{2}$ ratio in urine was less than 1 in 22 of 56 breast cancer patients treated with adjuvant tamoxifen while the ratio was greater than 1 in the other 34 patients (Fig. 4). Interestingly, 14 of 22 patients $(64 \%)$ with a $\mathrm{P}_{3} / \mathrm{P}_{2}$ ratio of $<1$ demonstrated hepatic steatosis (liver/spleen ratio of CT values in Hounsfield units $<0.9$ ) whereas only 4 of 30 patients (13\%) with a $\mathrm{P}_{3} / \mathrm{P}_{2}$ ratio $>1$ demonstrated hepatic steatosis. Fisher's exact test revealed that a 
Pretreatment

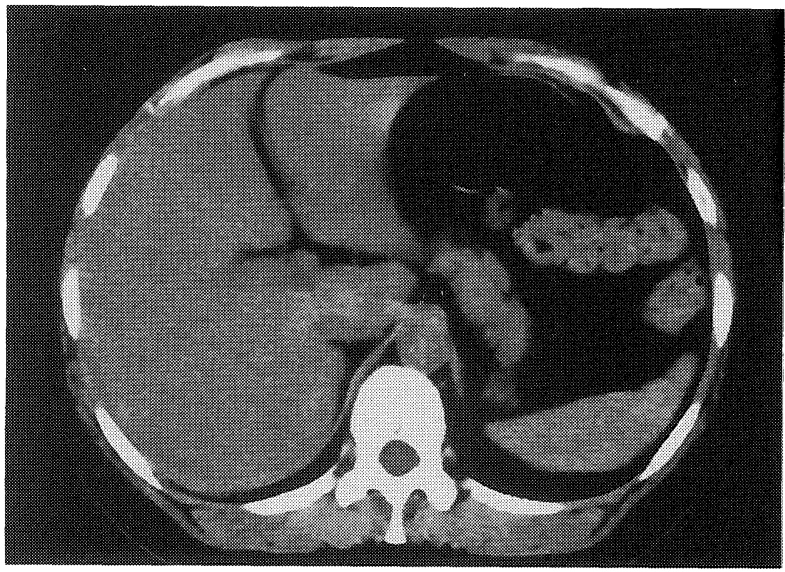

2 months later

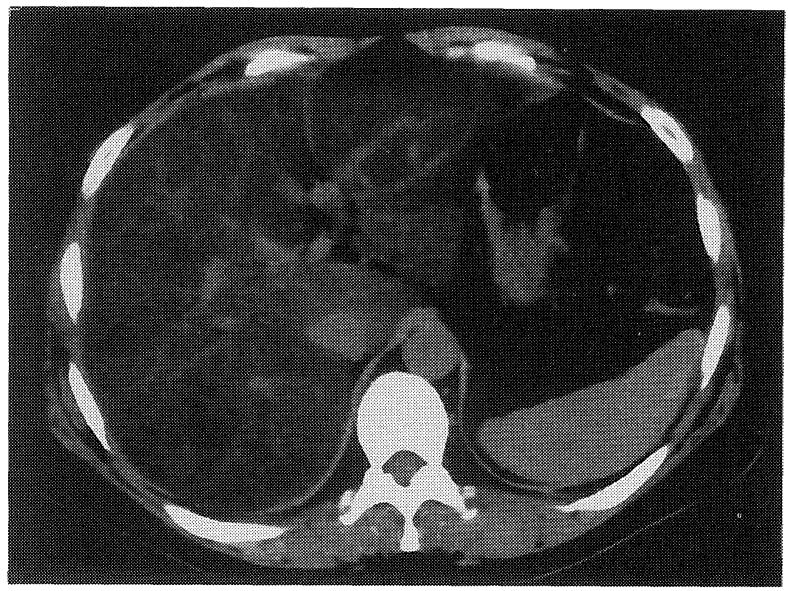

10 months later

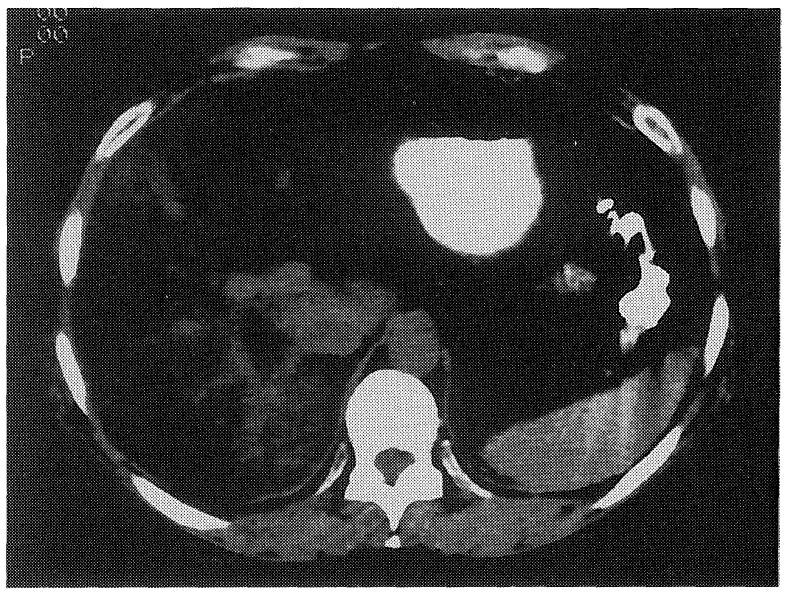

Figure 1. Sequential changes of computed tomography of an early breast cancer patient treated with adjuvant tamoxifen. The ratio of hepatic CT number to splenic CT number (liver/spleen ratio) rapidly and progressively decreased.
Liver/Spleen ratio

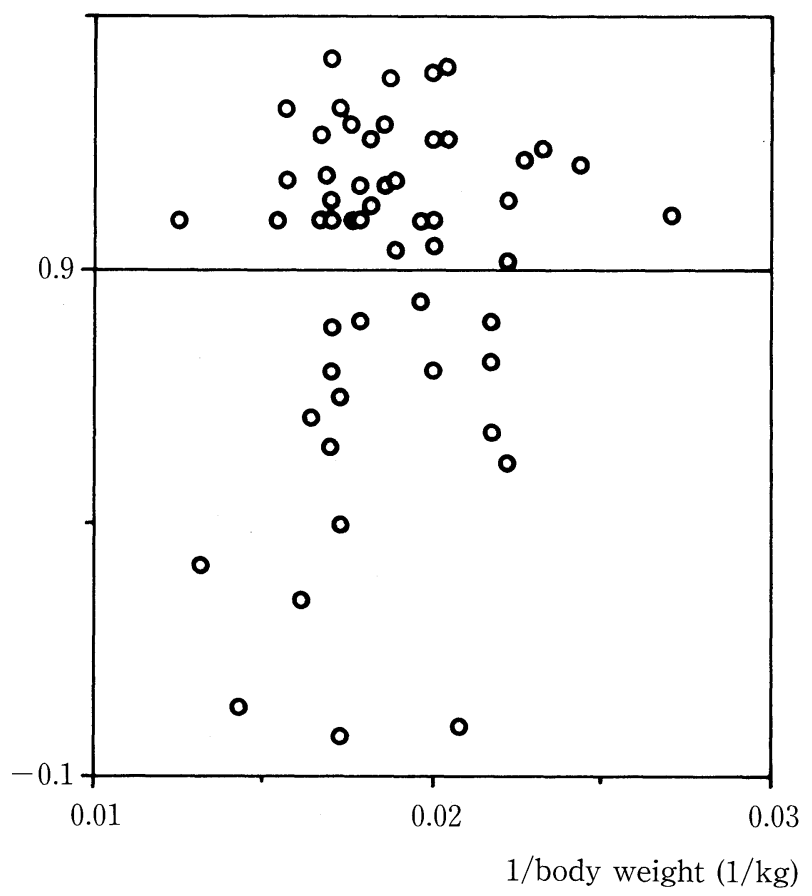

Figure 2. The relation between the inverse of body weight and the liver/spleen ratio. There was no significant difference in the dose of tamoxifen/body weight between patients who developed hepatic steatosis and who did not. This observation may exclude the possibility that the development of hepatic steatosis is due to excessive administration of tamoxifen.

$\mathrm{P}_{3} / \mathrm{P}_{2}$ ratio of $<1$ was related to a liver/spleen ratio of $<0.9$ $(\mathrm{p}<0.0001)$. These observations revealed that the cut-off value of 1.0 in the $\mathrm{P}_{3} / \mathrm{P}_{2}$ ratio was good for distinguishing the two patterns of hepatic response to adjuvant tamoxifen therapy. Indeed, 10 of 11 patients with $\mathrm{BMI}>23.6$ and $\mathrm{P}_{3} / \mathrm{P}_{2}$ ratio $<1$ suffered from hepatic steatosis (Fig. 5).

Kruskal-Wallis test revealed that $\mathrm{BMI}$ and $\mathrm{P}_{3} / \mathrm{P}_{2}$ ratios were not correlated to the severity of hepatic steatosis $(\mathrm{p}=0.322$ and 0.124 , respectively) suggesting that the severity of hepatic steatosis is determined by other undefined factors.

\section{A representative clinical course}

Figure 6 shows a representative clinical course among 10 tamoxifen-induced NASH patients treated with bezafibrate, whose body weight remained unchanged during the 5-year treatment with tamoxifen. All of these patients revealed an evident recovery of hepatic CT number within 10 months and some of these patients underwent liver biopsy to confirm these findings. These findings are in agreement with our previous report (18). AST and ALT levels were elevated mildly in the early phase of tamoxifen-induced hepatic steatosis, while $\gamma$-GTP level remained normal. Administration of bezafibrate attenuated elevated AST and ALT levels in the first several months, and an 
Liver/Spleen ratio

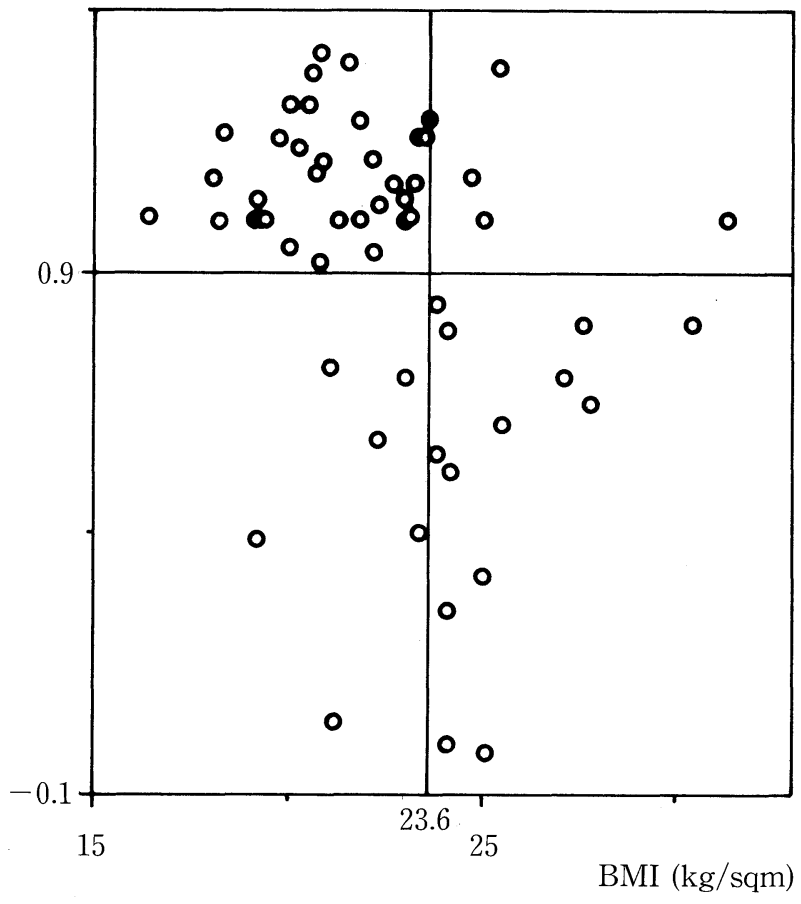

Figure 3. The relation between body mass index and the liver/ spleen ratio. Body mass index $>23.6 \mathrm{~kg} / \mathrm{sqm}$ was a significant risk factor for the development of substantial hepatic steatosis $(\mathbf{p}<0.0001)$. BMI: body mass index.

evident recovery in CT was also noticed (liver/spleen ratio: from -0.26 to 0.94 ) (Fig. 7). In this case, administration of bezafibrate ( $400 \mathrm{mg} /$ day) was interrupted because of the appearance of mild myalgia. As fat accumulated in the liver again (liver/spleen ratio 0.21) and transaminase levels relapsed within six months, she was treated with a low dose of fenofibrate (150 $\mathrm{mg}$ /day) thereafter. Myalgia was not noted and AST and ALT levels rapidly normalized soon after the cessation of the 5-year treatment of adjuvant tamoxifen (Fig. 6).

$\mathrm{P}_{3} / \mathrm{P}_{2}$ ratio was 1.57 before treatment, but decreased to 0.86 during adjuvant tamoxifen. Despite coadministration of bezafibrate, the value remained as low as 0.68 . Serum estradiol level was $48.3 \mathrm{pg} / \mathrm{ml}$ at the beginning of adjuvant tamoxifen, but remained $<10 \mathrm{pg} / \mathrm{ml}$ during adjuvant tamoxifen.

Figure 5. The relation between the body mass index and the pregnanetriol/pregnanediol ratio. Ten of 11 patients with BMI $>23.6$ and the pregnanetriol/pregnanediol ratio $<1$ suffered from hepatic steatosis (the liver/spleen ratio $<0.9$ ). Closed circles: patients with the liver/spleen ratio $<0.9$, Open circles: patients with the liver/spleen ratio $>0.9, \mathrm{BMI}$ : body mass index, $\mathbf{P}_{3} / \mathbf{P}_{2}$ ratio: pregnanetriol/pregnanediol ratio.

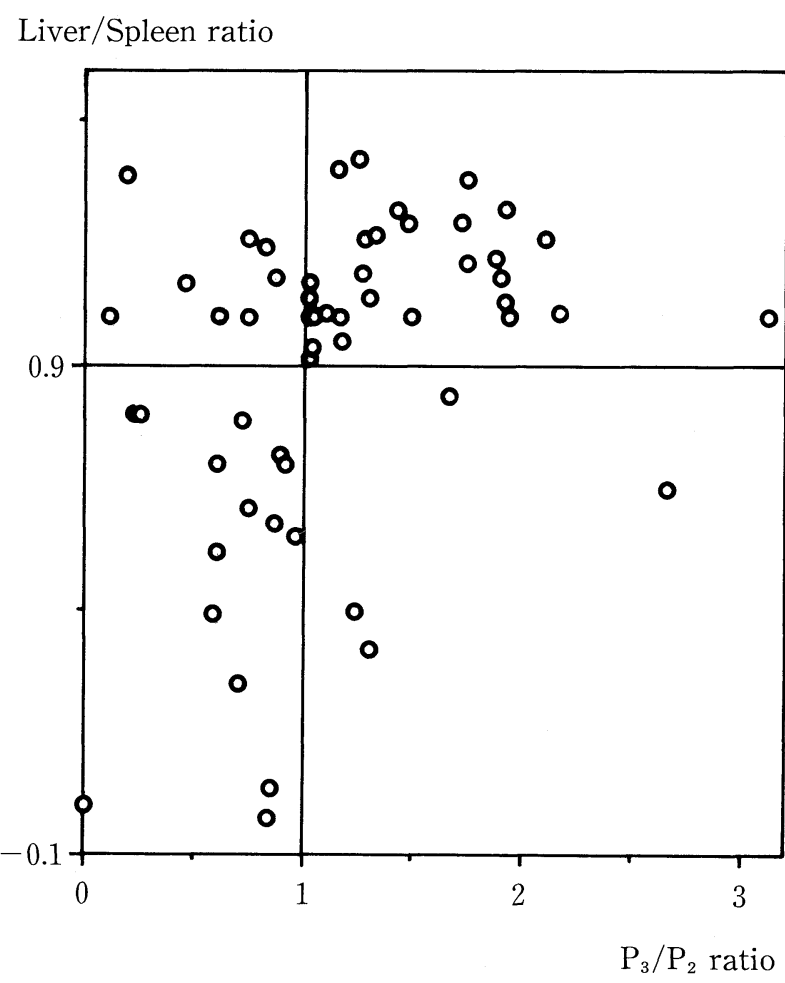

Figure 4. The relation between the pregnanetriol/pregnanediol ratio and the liver/spleen ratio. The liver/spleen ratio $<0.9$ was closely related to the pregnanetriol/pregnanediol ratio $<1.0$ (p<0.0001). $\mathbf{P}_{3} / \mathbf{P}_{2}$ ratio: pregnanetriol/pregnanediol ratio.

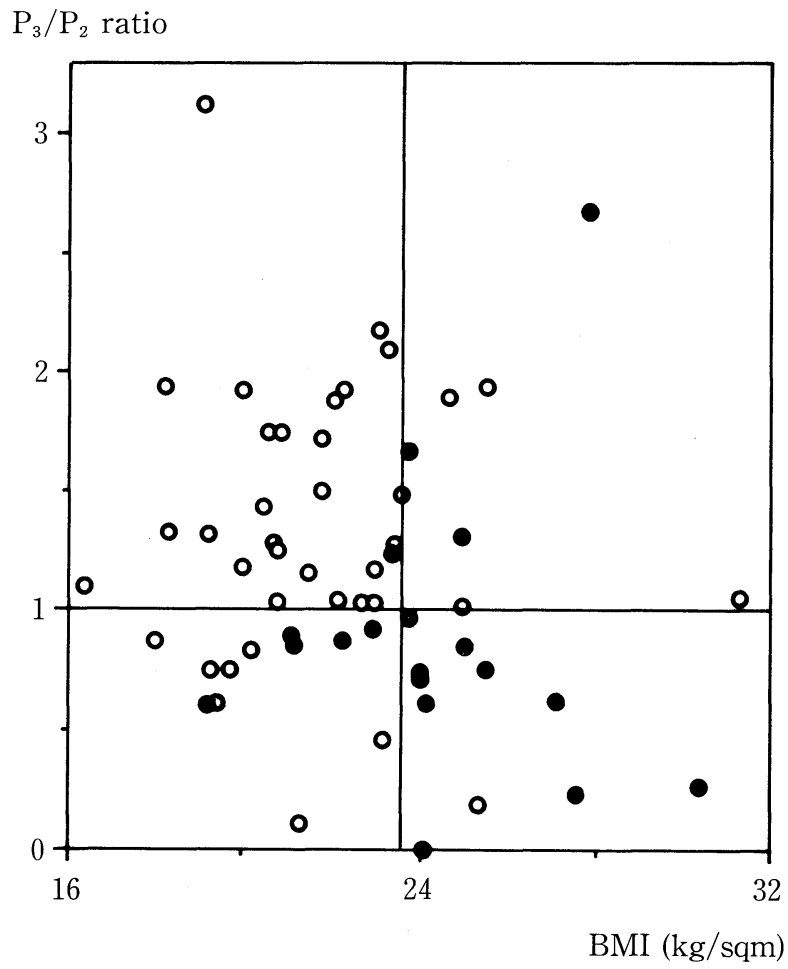




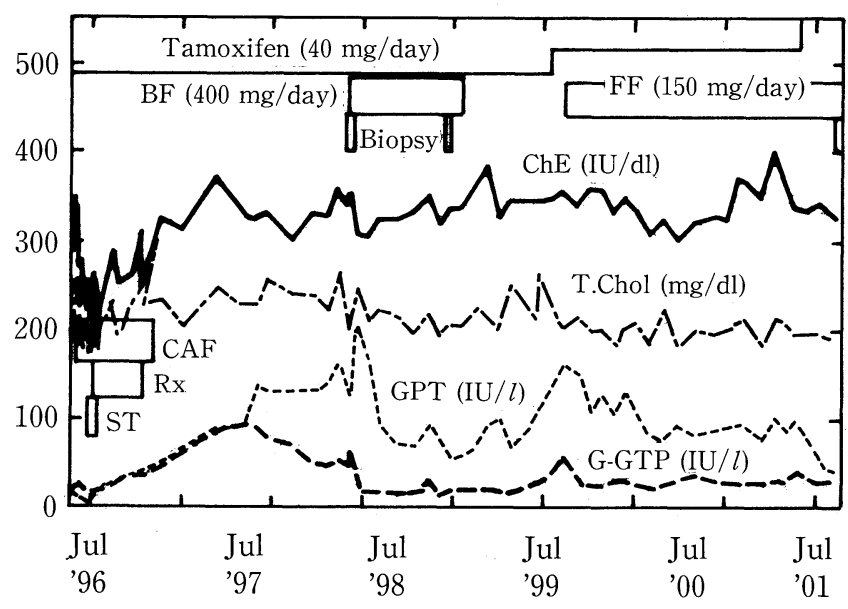

Figure 6. Clinical course of a representative patient successfully treated with adjuvant tamoxifen for 5 years. Sequential changes of computed tomography of this patient are shown in Fig. 7. The details of her clinical course are described in the text.

\section{Discussion}

The association of alcohol with liver damage is well established. Livers are enlarged due to severe fatty change in early stages. Pathological features in alcoholic liver disease are characterized by inflammatory changes and hepatocyte necrosis with the alcoholic hyaline of Mallory and various extents of liver fibrosis (21). During the 1980s, very similar pathological findings of the liver were recognized in nonalcoholics, who progressed to liver cirrhosis latently. Typical examples were observed as a frequent complication of jejunoileostomy for morbidly obese patients and as adverse reactions to medicine $(9,22)$. Since this disease entity was defined pathologically irrespective of the etiology, either fatty liver hepatitis or nonalcoholic steatohepatitis (NASH) was used to describe "the pathological and clinical features of non-alcoholic disease of the liver associated with the pathological features most commonly seen in alcoholic liver disease itself" (23). A variety of therapeutic approaches have been reported, but the consensus has not been reached.

As tamoxifen is an evident risk of NASH, we thought it important to elucidate the mechanisms involved in the rapid progression of hepatic steatosis during adjuvant tamoxifen and revealed that fatty acid $\beta$-oxidation could be profoundly impaired in estrogen deficiency using ArKO mouse as a model (7). However, the mechanism remained undefined why serum estradiol concentration frequently decreases in patients treated with tamoxifen and results in amenorrhea during adjuvant tamoxifen (24). Here, we proposed that estrogen might be involved in the feed forward mechanism of estrogen synthesis, because a $\mathrm{P}_{3} / \mathrm{P}_{2}$ ratio of $<1.0$ in urine was significantly related to a liver/spleen ratio of $<0.9$ (Fig. 4 ).

$\mathrm{P}_{2}$ is the major degradate of progesterone and $\mathrm{P}_{3}$ is the major degradate of 17-hydroxyprogesterone in urine. Therefore,

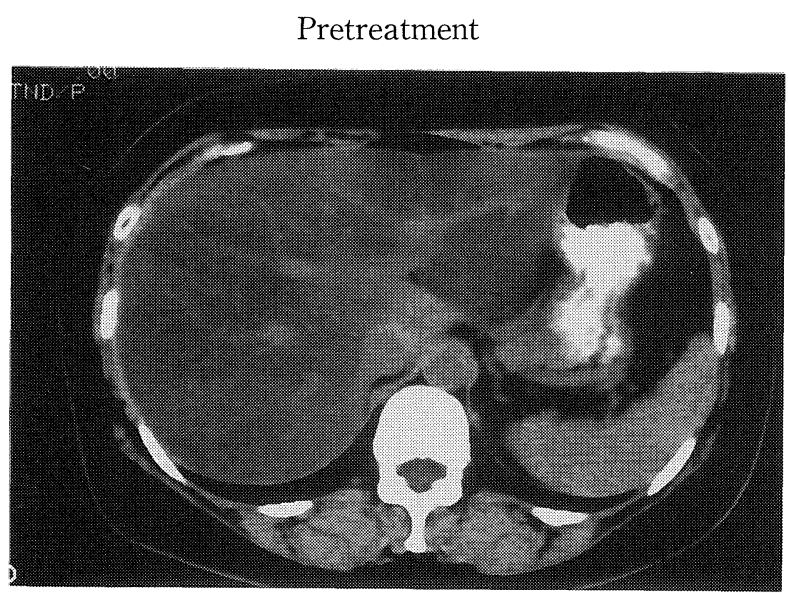

after treatment

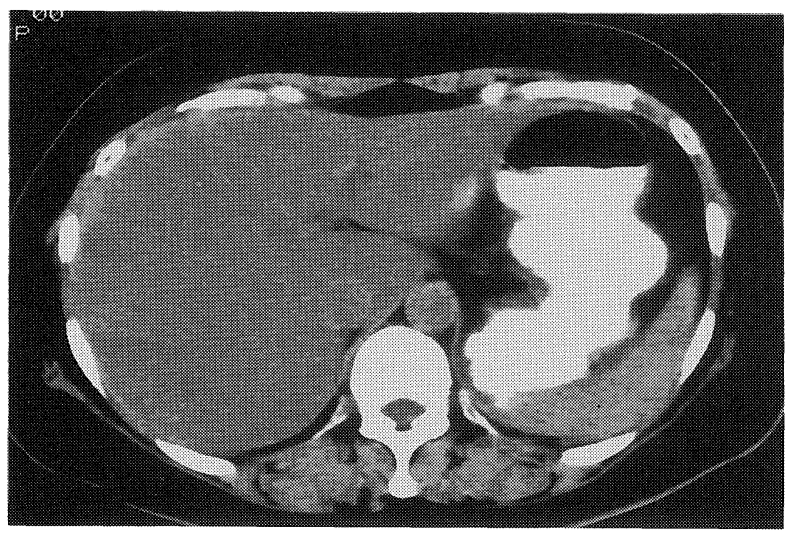

Exacerbation

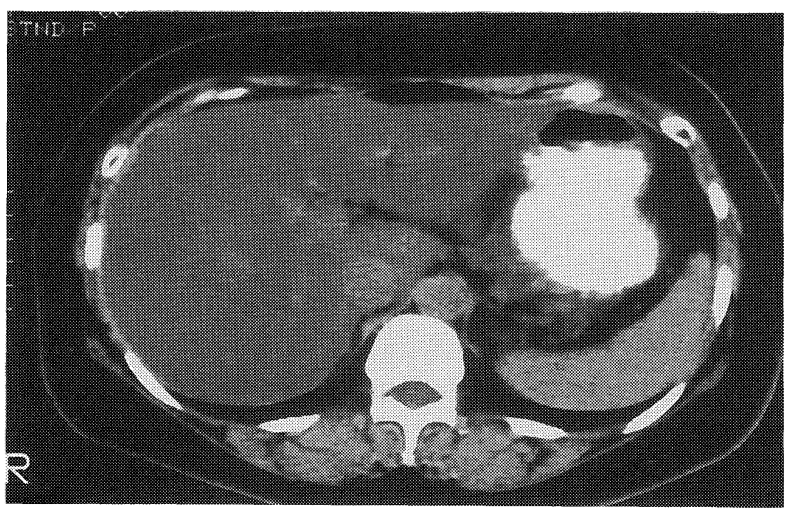

Figure 7. Sequential changes of computed tomography of a patient treated with bezafibrate. Her clinical course is described in the text and summarized in Fig. 6. Bezafibrate successfully eliminated accumulated triglycerides from her liver, but fat accumulated rapidly again after the cessation of bezafibrate.

$\mathrm{P}_{3} / \mathrm{P}_{2}$ ratio $<1.0$ could be a marker of reduced CYP17 $\alpha$ activity which catalyzes the hydroxylation of progesterone to 17hydroxyprogesterone (25). Because CYP17 $\alpha$ is the essential enzyme in the process of estrogen synthesis and is expressed 
in several steroidogenic tissues including adrenal cortex and ovary, these observations suggest that a sufficient blockade of fatty acid $\beta$-oxidation in the liver is closely related to a blockade of estrogen synthesis in the ovary. Indeed, we showed that the serum estrogen level was unusually low (undetectable level) in 48 of 56 premenopausal women treated with tamoxifen in this study. As the greatest concern of breast cancer patients and physicians is a sufficient blockade of the estrogen-mediated signaling pathway, we propose again that a $\mathrm{P}_{3} / \mathrm{P}_{2}$ ratio of $<1$ and/or a liver/spleen ratio of $<0.9$ could be markers of a blockade of estrogen synthesis and estrogen-mediated signaling pathways in vivo. This may be one reason why adjuvant tamoxifen is so effective in estrogenreceptor-positive breast cancer.

Here, we described a representative clinical feature of tamoxifen-induced NASH and suggested the administration of a peroxisome proliferator as one possible compensatory approach to prevent the progression of NASH. Bezafibrate was very effective since the extent of hepatic steatosis was improved substantially as shown in Fig. 7, but unfortunately the patient complained of mild myalgia in her extremities. There was no increase in serum level of creatine phosphokinase, but administration of bezafibrate was interrupted suddenly as rhabdomyolysis is a rare but serious complication associated with bezafibrate. We continued to administer tamoxifen and fat rapidly accumulated in the liver again. Because transaminase levels relapsed within six months, she was co-administered with a low dose of fenofibrate $(150 \mathrm{mg} /$ day $)$ thereafter.

We were not sure if fenofibrate was applicable in this case, because fenofibrate induces a slight increase in AST and ALT in some occasions. Fortunately myalgia was not noted in this case and serum levels of AST and ALT gradually dropped in response to fenofibrate. Interestingly soon after the cessation of 5-year treatment of adjuvant tamoxifen, AST and ALT levels rapidly normalized (Fig. 6). These observations confirmed that direct stimulation of peroxisome proliferator activated receptor- $\alpha$ can effectively activate $\beta$-oxidation of fatty acids in hepatocytes and improve massive hepatic steatosis dramatically in humans as observed in ArKO mice (19).

We do not recommend fibrates for all patients with hepatic steatosis, because the effects of fibrates on breast cancer and hepatic fibrosis remain to be elucidated. Therefore, we tentatively recommend administration of fibrate in combination with tamoxifen only for nonobese patients suffering from estrogen receptor-positive breast cancer and tamoxifen-induced NASH.

Acknowledgements: This work was partially supported by a grant from the Japanese Association of Medical Sciences and by a Grant-in-Aid for Scientific Research (C) 13670524 from the Japanese Ministry of Education, Science, Sports and Culture to TS.

\section{References}

1) Early Breast Cancer Trialists' Collaborative Group. Systemic treatment of early breast cancer by hormonal, cytotoxic, or immune therapy. 133 randomised trials involving 31,000 recurrences and 24,000 deaths among
75,000 women. Lancet 339: 71-85, 1992.

2) Fornander T, Rutqvist LE, Cedermark B, et al. Adjuvant tamoxifen in early breast cancer: occurrence of new primary cancers. Lancet 1: 117120, 1989.

3) Brun LD, Gagne C, Rousseau C, Moorjani S, Lupien PJ. Severe lipemia induced by tamoxifen. Cancer 57: 2123-2126, 1986.

4) Ogawa $Y$, Murata $Y$, Nishioka A, Inomata T, Yoshida S. Tamoxifen-induced fatty liver in patients with breast cancer. Lancet 351: 725, 1998 (letter).

5) Saibara T, Onishi S, Ogawa Y, Yoshida S, Enzan H. Non-alcoholic steatohepatitis. Lancet 354: 1299-1300, 1999 (letter).

6) Kato K, Takayama T, Katada N, et al. Evaluation of computed tomography in the diagnosis of liver diseases. Acta Hepatol Jpn 21: 1340-1351, 1980.

7) Nemoto Y, Toda K, Ono M, et al. Altered constitutive expression of fatty acid-metabolizing enzymes in aromatase-deficient (ArKO) mice. J Clin Invest 105: 1819-1825, 2000.

8) Toda K, Takeda K, Okada T, et al. Targeted disruption of the aromatase $\mathrm{P} 450$ gene (Cyp19) in mice and their ovarian and uterine responses to 17ß-oestradiol. J Endocrinol 170: 99-111, 2001.

9) James O, Day C. Non-alcoholic steatohepatitis: another disease of affluence. Lancet 353: 1634-1636, 1999.

10) Day CP, James OF. Hepatic steatosis: innocent bystander or guilty party? Hepatology 27: 1463-1466, 1998.

11) Wanless IR, Lentz JS. Fatty liver hepatitis (steatohepatitis) and obesity: an autopsy study with analysis of risk factors. Hepatology 12: 1106-1110, 1990.

12) Oien KA, Moffat D, Curry GW, et al. Cirrhosis with steatohepatitis after adjuvant tamoxifen. Lancet 353: 36-37, 1999 (letter)

13) Yang SQ, Lin HZ, Lane MD, Clemens M, Diehl AM. Obesity increases sensitivity to endotoxin liver injury: implications for the pathogenesis of steatohepatitis. Proc Natl Acad Sci USA 94: 2557-2562, 1997.

14) Day CP, James OF. Steatohepatitis: a tale of two "hits"? Gastroenterology 114: 842-845, 1998 (editorial).

15) Gail MH, Constantino JP, Bryant J, et al. Weighing the risks and benefits of tamoxifen treatment for preventing breast cancer. J Natl Cancer Inst 91: 1829-1846, 1999.

16) Murata $Y$, Ogawa $Y$, Saibara T, et al. Unrecognized hepatic steatosis and non-alcoholic steatohepatitis in adjuvant tamoxifen for breast cancer patients. Oncol Rep 7: 1299-1304, 2000.

17) Hamada N, Ogawa $Y$, Saibara $T$, et al. Toremifene-induced fatty liver and NASH in breast cancer patients with breast-conservation treatment. Intern J Oncol 17: 1119-1123, 2000.

18) Saibara T, Onishi S, Ogawa Y, Yoshida S, Enzan H. Bezafibrate for tamoxifen-induced non-alcoholic steatohepatitis. Lancet 353: 1802, 1999 (letter).

19) Yoshikawa T, Toda K, Nemoto Y, et al. Aromatase-deficient (ArKO) mice are retrieved from severe hepatic steatosis by peroxisome proliferator administration. Hepatol Res 2002 (in press).

20) Tsuboi N, Ogawa Y, Inomata T, et al. Changes in the findings of dynamic MRI by preoperative CAF chemotherapy for patients with breast cancer of stage II and III. Oncol Rep 6: 727-732, 1999.

21) Sherlock S, Dooley J. Alcohol and the Liver. in: Diseases of the Liver and Biliary System. 10th ed. Sherlock S, Dooley J, Eds. Blackwell Science Ltd, Oxford, United Kingdom, 1997: 385-404.

22) Berson A, De Beco V, Lețeron P, et al. Steatohepatitis-inducing drugs cause mitochondrial dysfunction and lipid peroxidation in rat hepatocytes. Gastroenterology 114: 764-774, 1998.

23) Ludwig J, Viggiano TR, McGill DB, Oh BJ. Nonalcoholic steatohepatitis: Mayo clinic experiences with a hitherto unnamed disease. Mayo Clin Proc 55: 434-438, 1980.

24) Bianco AR, Del Mastro L, Gallo C, et al. Prognostic role of amenorrhea induced by adjuvant chemotherapy in premenopausal patients with early breast cancer. Br J Cancer 63: 799-803, 1991.

25) Miller WL. Molecular biology of steroid hormone synthesis. Endocr Rev 9: $295-318,1988$. 